\title{
Analysis of the Risk Factors of Upper Limb Lymphedema after Breast Cancer Surgery and Nursing Care
}

\author{
Linlin Qian* \\ Yancheng's First People's Hospital, Yancheng 224000, Jiangsu Province, China \\ *Corresponding author: Linlin Qian, 152147976@qq.com
}

\begin{abstract}
Objective: To evaluate and analyze the influencing factors of upper limb lymphedema after breast cancer surgery, and to study effective nursing intervention measures. Methods: 500 cases of early breast cancer patients from October 2017 to December 2020 were selected, all patients underwent surgical intervention, retrospectively analyzed the basic clinical data of patients, and statistically analyzed the influencing factors of upper limb lymphedema. All patients with upper extremity lymphedema received high-quality nursing intervention, and the specific nursing effect was analyzed. Results: Logistic regression analysis showed that the risk factors of upper limb lymphedema after breast cancer surgery included hypertension, postoperative upper limb functional exercise, delayed healing of incision, radiotherapy and so on. After nursing intervention, the patients' elbow $10 \mathrm{~cm}$, elbow $10 \mathrm{~cm}$, wrist size value and VAS score were better than those before nursing $(\mathrm{P}<0.05)$. The quality of life score of patients after nursing intervention was significantly better than that before nursing $(\mathrm{P}<0.05)$. Conclusion: Hypertension, postoperative upper limb functional exercise, delayed healing of incision, radiotherapy and other factors can induce upper limb lymphedema after breast cancer surgery. Effective nursing intervention can alleviate the condition of patients with upper limb lymphedema and improve their quality of life, which is worthy of comprehensive promotion.
\end{abstract}

Keywords: Mammary cancer; Lymphedema of upper extremity; Occurrence factors; Nursing

Publication date: September 2021; Online publication: September 30, 2021

\section{Introduction}

Upper limb lymphedema has no multiple complications after breast cancer surgery, which can lead to limb movement dysfunction, and can also lead to negative emotions of patients, which seriously affect the prognosis. Therefore, it is necessary to deeply analyze the influencing factors of upper limb lymphedema after breast cancer surgery, and explore reasonable and effective nursing intervention programs. Therefore, to reduce the incidence of upper limb lymphedema after breast cancer surgery and improve the prognosis of such diseases Treatment effect of the disease ${ }^{[1-2]}$. This study summarized the basic clinical data of patients in our hospital, studied and systematically analyzed the high-risk factors of upper limb lymphedema after breast cancer surgery, and studied the effective nursing intervention program.

\section{Material and methods}

\subsection{General information}

500 patients with early breast cancer were selected from October 2017 to December 2020. All patients were diagnosed with breast cancer by comprehensive examination. The estimated survival time was more than three months. There were no cognitive and language disorders, non-bilateral breast cancer and recurrent 
breast cancer, and no organ metastasis. They agreed to participate in this study. The basic clinical data of 500 patients were collected and analyzed. The range of age ranged from 24 to 65 years old, with an average of $(45.38 \pm 3.79)$ years old, including 248 cases on the left side, 252 cases on the right side, 104 cases in stage I, 248 cases in stage II and 148 cases in stage III.

\subsection{Methods}

The data of age, body mass index, lesion location, disease stage, axillary lymph node dissection, tumor volume, radiotherapy, chemotherapy and endocrine therapy, postoperative upper limb functional exercise, delayed healing, combined hypertension and infection were analyzed to determine the influencing factors of upper limb lymphedema after breast cancer surgery.

A total of 62 patients with lymphedema of upper limbs were treated with high-quality nursing intervention.

\subsubsection{Skin tissue care}

The nursing staff told the patients to wear soft and loose clothes to avoid skin tissue friction and scratch. If the patients have skin redness, swelling, heat, pain and ulceration, they need to inform the doctor in time and follow the doctor's advice for anti-infection treatment.

\subsubsection{Comprehensive functional exercise}

Postoperative functional exercise should be completed step by step to promote the disappearance of edema and improve the function of shoulder joint. Early rehabilitation exercise (within two weeks after operation).

(1) The first section was clenching and loosening (24 hours after operation)

(2) The second segment was wrist movement, wrist movement up and down, combined with internal and external rotation ( $48 \mathrm{~h}$ after operation).

(3) The third segment was forearm movement, forearm flexion and extension (3 days after operation).

(4) The fourth segment was elbow movement, with the waist as the support point, the arm was raised properly, placed in front of the contralateral chest, and completed alternately on both sides (5 days after operation).

(5) In the fifth segment, the healthy side held the affected elbow and raised it to the chest (7 days after operation).

(6) In the sixth segment, the shoulder was relaxed and rotated forward and backward (9 days after operation).

(7) The seventh segment was upper arm movement, and the upper arm was raised parallel to the ground (10 days after operation).

(8) The eighth segment was neck movement, neck rotation in different directions, hands akimbo (11 days after operation).

(9) The ninth section is body rotation. The arm swung forward and backward, and the upper body swung left and right (11 days after operation).

(10) The tenth segment was shoulder lifting. The healthy side held the affected wrist, raised it to the chest, and stretched and stretched forward (14 days after operation). 


\subsubsection{Lymphedema massage}

The nurses fully moved the wrist joint, elbow joint and fingers of the patients, and carried out acupoint massage on the upper limb lymph nodes to guide the patients to complete the abduction and adduction related exercises of elbow joint and shoulder joint, so as to alleviate the edema.

\subsubsection{Dietary guidance and psychological counseling}

Postoperative nursing staff should formulate healthy diet for patients, inform patients that the main principle of daily diet is low fat and low salt, increase the intake of calories, vitamins and protein, ensure that the daily diet is light and easy to digest, and avoid eating spicy food. Some patients with upper limb lymphedema after breast cancer surgery have serious negative emotions. Therefore, nursing staff need to pacify them, inform them of the pathogenesis and treatment plan of such complications, correct their misconceptions, give them full encouragement, and introduce the case data of successful patients, so that they can actively cooperate with the treatment intervention, thereby to promote the recovery of the disease.

\subsection{Evaluation criteria}

Logistic regression analysis was used to evaluate the influencing factors of upper limb lymphedema after breast cancer surgery.

\section{4, Statistical methods}

SPSS 23.0 software was used to calculate all kinds of data, the measurement data was $(\overline{\mathrm{x}} \pm \mathrm{s})$, the test method was t, the count data was $(\%)$, the test method was $X^{2}$, if $P<0.05$, there were differences between groups.

\section{Results}

\subsection{Analysis of influencing factors of upper limb lymphedema after breast cancer surgery}

Logistic regression analysis showed that the risk factors of upper limb lymphedema after breast cancer surgery included hypertension, postoperative upper limb functional exercise, delayed healing of incision, radiotherapy, etc. (Table 1.).

Table 1. Analysis of influencing factors of upper limb lymphedema after breast cancer surgery

\begin{tabular}{|c|c|c|c|c|}
\hline Group & $95 \% \mathrm{CI}$ & SE & Wald & $\boldsymbol{\beta}$ \\
\hline $\begin{array}{l}\text { Complicated with } \\
\text { hypertension }\end{array}$ & $(1.135,19.127)$ & 0.815 & 4.489 & 1.722 \\
\hline $\begin{array}{l}\text { Postoperative upper } \\
\text { limb functional } \\
\text { exercise }\end{array}$ & $(0.048,0.866)$ & 0.765 & 4.669 & -1.646 \\
\hline $\begin{array}{c}\text { Delayed healing of } \\
\text { incision after operation }\end{array}$ & $(2.914,13.085)$ & 1.197 & 3.529 & 2.138 \\
\hline Radiotherapy & $(1.962 .32 .015)$ & 0.773 & 8.004 & 2.196 \\
\hline
\end{tabular}




\subsection{Comparative analysis of nursing intervention before and after the elbow $10 \mathrm{~cm}$, elbow $10 \mathrm{~cm}$, wrist size and VAS score}

After nursing intervention, the patients' elbow $10 \mathrm{~cm}$, elbow $10 \mathrm{~cm}$, wrist size and VAS score were better than before nursing $(P<0.05)$ (Table 2.).

Table 2. Comparative analysis of upper arm, forearm difference and VAS score ( $\overline{\mathrm{x}} \pm \mathrm{s})$ before and after nursing intervention

\begin{tabular}{ccccc}
\hline Group & $\begin{array}{c}\mathbf{1 0} \text { cm above } \\
\text { elbow }(\mathbf{C M})\end{array}$ & $\begin{array}{c}\mathbf{1 0} \text { cm below } \\
\text { elbow }(\mathbf{C M})\end{array}$ & Wrist $(\mathbf{C M})$ & VAS score \\
\hline Before nursing $(\mathrm{n}=62)$ & $20.73 \pm 0.15$ & $18.99 \pm 0.25$ & $10.27 \pm 0.15$ & $3.96 \pm 1.28$ \\
After nursing $(\mathrm{n}=62)$ & $18.18 \pm 0.49$ & $17.72 \pm 0.16$ & $9.12 \pm 0.06$ & $1.45 \pm 0.27$ \\
T value & 39.182 & 33.690 & 56.049 & 15.107 \\
P value & 0.000 & 0.000 & 0.000 & 0.000 \\
\hline
\end{tabular}

2.3 Quality of life scores before and after nursing intervention were compared and analyzed

The quality of life score after nursing intervention was significantly better than that before nursing intervention $(\mathrm{P}<0.05)$ (Table 3.).

Table 3. Quality of life score $(\overline{\mathrm{x}} \pm \mathrm{s})$ before and after nursing intervention

\begin{tabular}{ccccc}
\hline Group & Sociology & Emotion & Physiology & Function \\
\hline Before nursing $(\mathrm{n}=62)$ & $17.38 \pm 5.24$ & $17.44 \pm 5.16$ & $18.31 \pm 5.65$ & $10.36 \pm 2.97$ \\
After nursing $(\mathrm{n}=62)$ & $21.79 \pm 4.95$ & $22.19 \pm 5.83$ & $22.86 \pm 6.33$ & $14.77 \pm 4.05$ \\
t value & 4.817 & 4.803 & 4.222 & 6.914 \\
P value & 0.000 & 0.000 & 0.000 & 0.0 \\
\hline
\end{tabular}

\section{Discussion}

The treatment of breast cancer can lead to the blocking of upper arm lymph reflux path, a large number of protein containing lymph components in the interstitial tissue, significantly increased filtration pressure, and decreased plasma protein content, resulting in a significant decrease in colloidal osmotic pressure, and induced capillary permeability, protein substances in the interstitial fluid cannot be effectively removed, lymph has a strong effect on the interstitial tissue Irritative effect, so that subcutaneous tissue fibrosis, lymph drainage limited continued to aggravate, and then the formation of lymphedema ${ }^{[3]}$.

To summarize and evaluate the data of this study, the influencing factors of upper limb lymphedema after breast cancer surgery mainly include hypertension, postoperative upper limb functional exercise, delayed healing of incision, radiotherapy and so on. The increase of blood pressure can lead to the increase 
of the amount of material in blood vessels to the outside, which can significantly increase the amount of lymph production in patients, and then form lymphedema in upper limbs ${ }^{[4]}$. The failure of upper limb motor function training after operation can slow down the recovery speed of limb blood supply and lymph circulation, and a large amount of lymph remains in interstitial cells, leading to upper limb lymphedema. At the same time, the delayed healing of incision can lead to the aggravation of lymphatic vessel injury, the obstruction of collateral circulation and lymphatic vessel reconstruction, and then induce upper extremity lymphedema. Radiotherapy can cause serious damage to the body, which can lead to lymphatic and vascular occlusion in the radiation field, muscle fibrosis, lymphatic and venous compression, and lymphedema ${ }^{[5-6]}$.

After the high-quality nursing intervention, the patients' elbow $10 \mathrm{~cm}$, elbow $10 \mathrm{~cm}$, wrist size, VAS score and quality of life score were better than those before the nursing intervention. High quality nursing of patients with upper extremity lymphedema as the core, nursing staff analysis of patients with disease characteristics, combined with their nursing service needs to develop various nursing interventions. Functional training can gradually restore the motor function of upper limbs, promote the return of lymph fluid to normal, form compensatory neovascularization of blood vessels and lymphatic vessels, significantly improve the living ability and motor ability of patients, and then improve their quality of life [7-8]. The massage of lymphedema of upper limbs is simple and noninvasive, and it can promote blood circulation and relieve edema by squeezing the deep tissue. At the same time, this study adds psychological nursing intervention measures, nurses give patients sufficient humanistic care, inform their disease-related knowledge, can maximize the improvement of patients' mental health, improve the degree of nursing cooperation, help to improve the prognosis.

In conclusion, hypertension, postoperative upper limb functional exercise, delayed healing of incision, radiotherapy and other factors can induce upper limb lymphedema after breast cancer surgery. Effective nursing intervention can alleviate the condition of patients with upper limb lymphedema and improve their quality of life, which is worthy of comprehensive promotion.

\section{Disclosure statement}

The author declares no conflict of interest.

\section{References}

[1] Chen WP, 2020, Clinical Effect of Targeted Nursing on Postoperative Upper Extremity Lymphedema in Breast Cancer Patients. Journal of Applied Clinical Nursing, 5(6): 40.

[2] Sun WW, Sun R, Dong LL, et al., 2020, The Effect of Extended Nursing Mode on the Prevention and Control of Lymphedema in Breast Cancer Patients based on the Theory of Power. Journal of Changchun University of Traditional Chinese Medicine, 36(5): 1034-1037.

[3] Payue ZS, Yang CF, Huang LH, et al., 2020, Study on the Effect of Bare Hand Lymphatic Drainage Technique and Rehabilitation Nursing in Lymphedema after Lower Extremity Trauma. Health Care Guide, 2020, (7):168-169.

[4] Wang J, 2020, Application of Nursing Quality Index Monitoring in the Prevention of Breast Cancer Related Upper Extremity Lymphedema Health Education. Family with Pregnant Treasure, 2(14):278.

[5] Ma L, 2020, Discussion on the Application of Pressure Nursing Intervention in the Nursing of Affected Limb Lymphedema after Breast Cancer Surgery. Health Vision, (9):125.

[6] Liu WL, 2020, Application of Rehabilitation Group Intervention Model in Postoperative Upper Extremity Lymphedema Rehabilitation Nursing of Breast Cancer Patients. Chinese Community Physician, 36(15): 145-146. 
[7] Zhang HY, 2020, Effect of Nursing Intervention on Upper Extremity Lymphedema Patients after Breast Cancer Surgery and its Impact on Quality of Life. Chinese and Foreign Women's Health Research, (11): 149-161.

[8] Hao RZ, Shen YH, Wang YX, et al., 2020, Clinical Analysis of Lymphedema after Breast Cancer Surgery. Health Vision, (22): 122-125. 